\title{
Structural and magnetic properties of irradiated $\mathrm{SiC}$
}

Yutian Wang ${ }^{1,4}$, Xuliang Chen ${ }^{2}$, Lin $\mathrm{Li}^{1,3}$, Artem Shalimov ${ }^{1}$, Wei Tong ${ }^{5}$, Slawomir Prucnal $^{1}$, Frans Munnik ${ }^{1}$, Zhaorong Yang ${ }^{2}$, Wolfgang Skorupa ${ }^{1}$, Manfred Helm ${ }^{1,4}$, Shengqiang Zhou ${ }^{1}$

1. Institute of Ion Beam Physics and Materials Research, Helmholtz-Zentrum Dresden-Rossendorf (HZDR), P.O. Box 510119, 01314 Dresden, Germany

2. Key Laboratory of Materials Physics, Institute of Solid State Physics, Chinese Academy of Sciences, Hefei 230031, People's Republic of China

3. Department of Physics and Electronics, School of Science, Beijing University of Chemical Technology, Beijing 100029, China

4. Technische Universität Dresden, 01062 Dresden, Germany

5. High Magnetic Field Laboratory, Hefei Institutes of Physical Science, Chinese Academy of Sciences, Hefei 230031, People's Republic of China

\section{Abstract:}

We present a comprehensive structural characterization of ferromagnetic $\mathrm{SiC}$ single crystals induced by $\mathrm{Ne}$ ion irradiation. The ferromagnetism has been confirmed by electron spin resonance and possible transition metal impurities can be excluded to be the origin of the observed ferromagnetism. Using X-ray diffraction and Rutherford backscattering/channeling spectroscopy, we estimate the damage to the crystallinity of $\mathrm{SiC}$ which mutually influences the ferromagnetism in SiC. 


\section{Introduction:}

Defect-induced ferromagnetism in materials which do not contain partially filled $3 d$ or $4 f$ electrons has recently entered the focus of condensed matter research [1]. It challenges the $m J$ paradigm for magnetism, where $m$ refers the local moment and $J$ stands for the interaction between the local moments. Experimentally, defect-induced ferromagnetism was observed in many materials, including graphite [2-5] and various oxides [6-12]. SiC single crystals are emerging as another candidate for this investigation and have been shown to be ferromagnetic after particle irradiation [13, 14] or after aluminum doping [15]. The magnetization in $\mathrm{SiC}$ is found to sensitively depend on the fluence of ion or neutron irradiation. Compared with other materials, $\mathrm{SiC}$ is commercially available at large scale with the microelectronic quality grade [16]. In this paper, we present a systematic structural investigation in correlation with the magnetic properties of $\mathrm{SiC}$ prepared by $\mathrm{Ne}$ ion irradiation. A possible $\mathrm{Fe}, \mathrm{Co}$ or $\mathrm{Ni}$ contamination in SiC is excluded by particle induced X-ray emission (PIXE) and by Auger electron spectroscopy (AES) measurements. The appearance of ferromagnetism has been confirmed by electron spin resonance (ESR) spectroscopy. Measurements of X-ray diffraction (XRD) and Rutherford backscattering/channeling spectroscopy $(\mathrm{RBS} / \mathrm{C})$ reveal the damage to the crystallinity of SiC which can extinguish the ferromagnetism in SiC.

\section{Experimental methods:}

The $6 \mathrm{H}$-SiC(0001) wafer purchased from KMT corporation (Hefei, China) is one-side polished and semi-insulating. The same wafer was cut into smaller pieces which were implanted with $\mathrm{Ne}$ ions at an energy of $140 \mathrm{keV}$. The ion fluences were $5 \times 10^{13}$, Page | 2 
$1 \times 10^{14}, 5 \times 10^{14}, 1 \times 10^{15} \mathrm{~cm}^{-2}$, and consequently the samples will be named as $5 \mathrm{E} 13$, $1 \mathrm{E} 14,5 \mathrm{E} 14$ and 1E15, respectively. Magnetometry was performed using a MPMS-XL magnetometer from Quantum Design. The ferromagnetic resonance was measured at $9.46 \mathrm{GHz}$ by an electron paramagnetic resonance spectrometer (Bruker ELEXSYS E500). PIXE was performed using $3 \mathrm{MeV}$ protons with a broad beam of $1 \mathrm{~mm}^{2}$, while AES was done by a scanning Auger electron spectrometer Microlab 310F (Fisons Instruments). In order to evaluate the crystalline variation after ion irradiation, synchrotron radiation XRD was carried out at the Rossendorf beamline (BM20) at the ESRF with an X-ray wavelength of $0.1078 \mathrm{~nm}$. As a complementary method, RBS/C with $1.7 \mathrm{MeV} \mathrm{He}^{+}$was used to quantitatively determine the ion-implantation-induced atomic disorder of the Si sublattice in $6 \mathrm{H}-\mathrm{SiC}$.

\section{Results and discussion:}
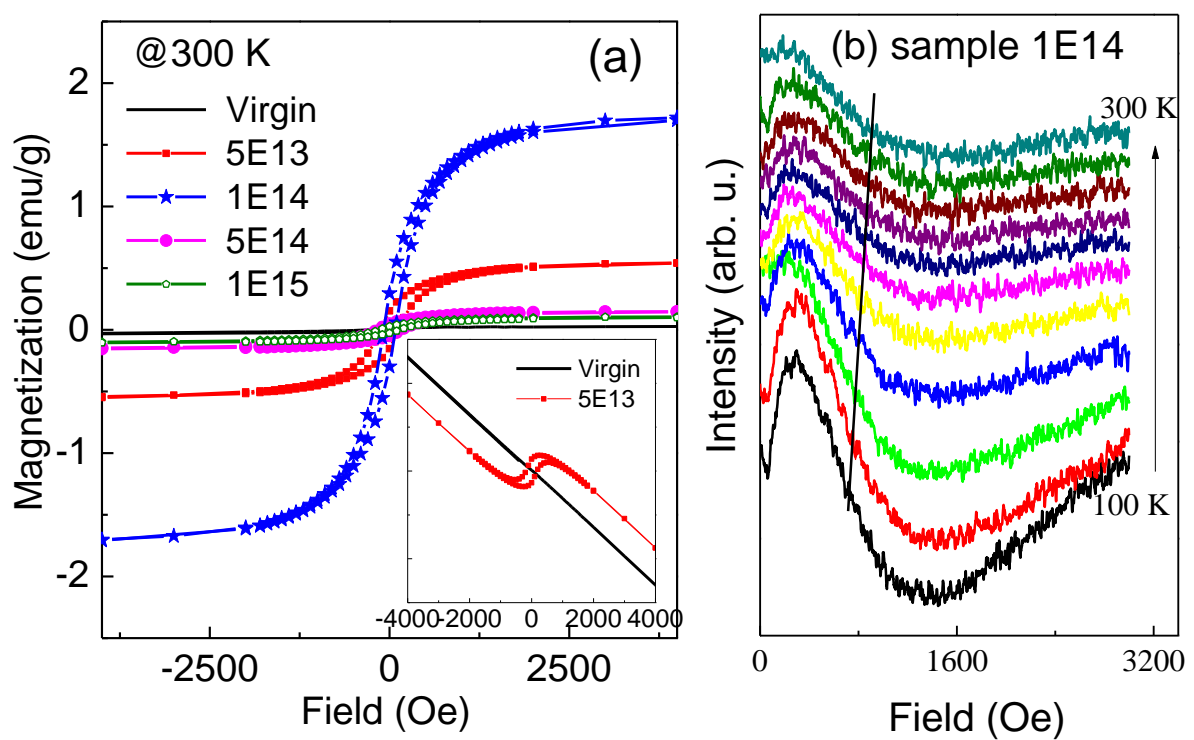

Fig. 1: (a) Magnetization vs. field measured at $300 \mathrm{~K}$ for all samples: the diamagnetic background from the substrate has been subtracted. The magnetization was calculated by assuming a thin layer of 460 $\mathrm{nm}$ thickness. The inset shows the magnetization measured for the virgin sample (black line) and 
sample $5 \mathrm{E} 13$ without subtracting the background. (b) ESR spectra of sample $1 \mathrm{E} 14$ at temperatures between 100 and $300 \mathrm{~K}$ : a broad resonance peak appears indicating ferromagnetic resonance. The black line indicates the resonance centers which slightly change with temperature.

\section{A. Magnetic properties}

The magnetic properties of $\mathrm{Ne}$ irradiated samples at different temperatures have been reported in our previous paper [13]. The inset of Fig. 1(a) shows the magnetization at $300 \mathrm{~K}$ measured for sample $5 \mathrm{E} 13$ and the virgin $\mathrm{SiC}$ from the same wafer. The virgin $\mathrm{SiC}$ only shows diamagnetism, while sample $5 \mathrm{E} 13$ presents a ferromagnetic hysteresis. Fig. 1(a) shows the magnetization at $300 \mathrm{~K}$ for all samples after subtracting the diamagnetic background. The saturation magnetization shows a nonlinear dependence with increasing Ne fluence (defect concentration) and reaches its maximum for sample $1 \mathrm{E} 14$.

The appearance of the ferromagnetism in ion irradiated $\mathrm{SiC}$ is further confirmed by ESR. We have measured virgin $\mathrm{SiC}$, sample $1 \mathrm{E} 14$ and one paramagnetic $\mathrm{SiC}$ in a broad field range. Only for sample 1E14, a broad ESR peak, and a shift of the center of the resonance at lower temperatures are clearly observed (Fig. 1b). Even at $300 \mathrm{~K}$, the ESR signal shows a very large shift from the free-electron position (around 3300 Oe), showing that the sample is ferromagnetic and has $T_{\mathrm{c}}$ above $300 \mathrm{~K}$. The $g$-factor increases slightly with decreasing temperatures.

\section{B. Scrutinizing possible magnetic contaminations}

For the investigation of defect-induced ferromagnetism, the crucial issue is to carefully examine if the ferromagnetic contamination is actually responsible for the Page | 4 
observed ferromagnetism. The magnetization [Fig. 1(a)] shows a clear dependence on the ion fluence, however the magnetic contaminations are expected to be random or to increase with ion fluence if they are due to ion implantation. We further applied PIXE and AES to scrutinize $\mathrm{Fe} / \mathrm{Co} / \mathrm{Ni}$ elements in our studied $\mathrm{SiC}$ specimens. Figure 2 shows the PIXE spectra for virgin $\mathrm{SiC}$ and $5 \mathrm{E} 13$. In the spectrum, the narrow peak is from Si K-line X-ray emission. The broad peak is due to the Bremsstrahlung background. No transition metal impurities were detected within the detection limit of around $1 \mu \mathrm{g} / \mathrm{g}$.

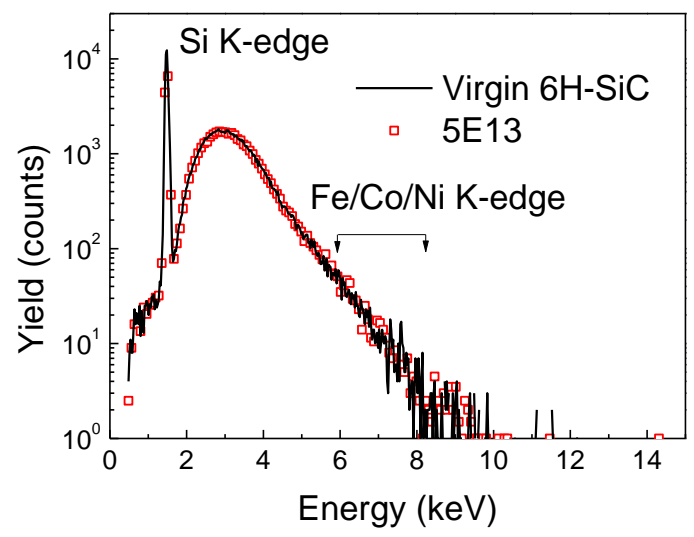

Fig. 2 PIXE spectrum for the virgin SiC wafer and a ferromagnetic sample. Within the detection limit, we do not observe any Fe, Co or Ni contamination.

To exclude contamination introduced during implantation and handling of the samples, we applied the surface sensitive AES method to the ferromagnetic pieces. For most elements, the detection limit of AES is in the range of $0.01-0.1$ at $\%$, which is around $10^{12}-10^{13}$ atom/ $/ \mathrm{cm}^{2}$ by assuming a detection depth of $2 \mathrm{~nm}$. We measured both the front and back sides for virgin and ferromagnetic $\mathrm{SiC}$ after $\mathrm{Ne}$ irradiation and did not observe any signal from $\mathrm{Fe} / \mathrm{Co} / \mathrm{Ni}$ within the detection limit (not shown). 


\section{Structural properties}

In order to correlate magnetization with structure, we performed XRD and RBS/C to check the crystalline deformation and defects.

Figure 3 depicts the $\theta-2 \theta$ scans at $\operatorname{SiC}(00012)$ recorded for $\mathrm{Ne}$ ion irradiated $6 \mathrm{H}$ SiC. A virgin sample is also shown for comparison. All curves show a main sharp diffraction peak at the diffraction angle $\theta=\theta_{\text {Bragg }}$ coming from the sample volum below the irradiated part. The diffraction peaks at the low angle side of the main Bragg peak, $\left(\theta<\theta_{\text {Bragg }}\right)$ are the characteristics of an expansion gradient of the lattice along SiC[0001]. The elastic strain in the near surface region $(\Delta d / d)$ can be deduced from the position of this satellite peak. Except for the largest fluence applied here (1E15), a fringe pattern is clearly visible. This feature is due to the presence of the non-homogeneous lattice expansion along the depth and arises from interferences between $x$-ray beams diffracted from regions with the same lattice spacing. The maximum value of the normal strain $(\Delta d / d)_{M}$ is estimated to be roughly proportional to the fluence. Furthermore, we also performed the corresponding reciprocal spacing mapping (RSM) around $\mathrm{SiC}(00012)$ (see Figure 4). Only a vertical streak coming from the irradiated region of the crystals is observed. No broadening in the horizontal direction (equivalent to a rocking curve) is measured as compared with that obtained from virgin crystals. These observations indicate that the defects are randomly distributed point defects or small defect clusters [18].

For the sample irradiated with the largest fluence of $1 \mathrm{E} 15 \mathrm{~cm}^{-2}$, the absence of the satellite peak shows that the near-surface region of this sample is heavily damaged, i.e. no more crystalline. Such a signal is consistent with a strongly defective crystalline lattice characterized by the presence of extended defects: amorphous 
clusters or layer [18]. The conclusions from XRD observation are fully consistent with the observation by positron annihilation spectroscopy, which reveals the clustering of vacancies [13].

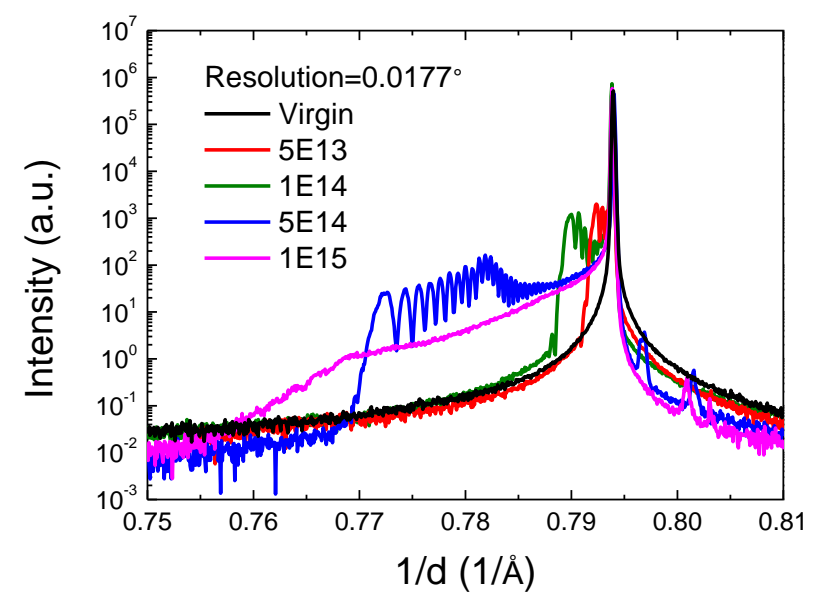

Fig. 3 XRD 2 $2 \theta / \theta$ scans of the virgin and $\mathrm{Ne}$ irradiated $6 \mathrm{H}-\mathrm{SiC}$ recorded in the vicinity of the $(00012)$ reflection. 

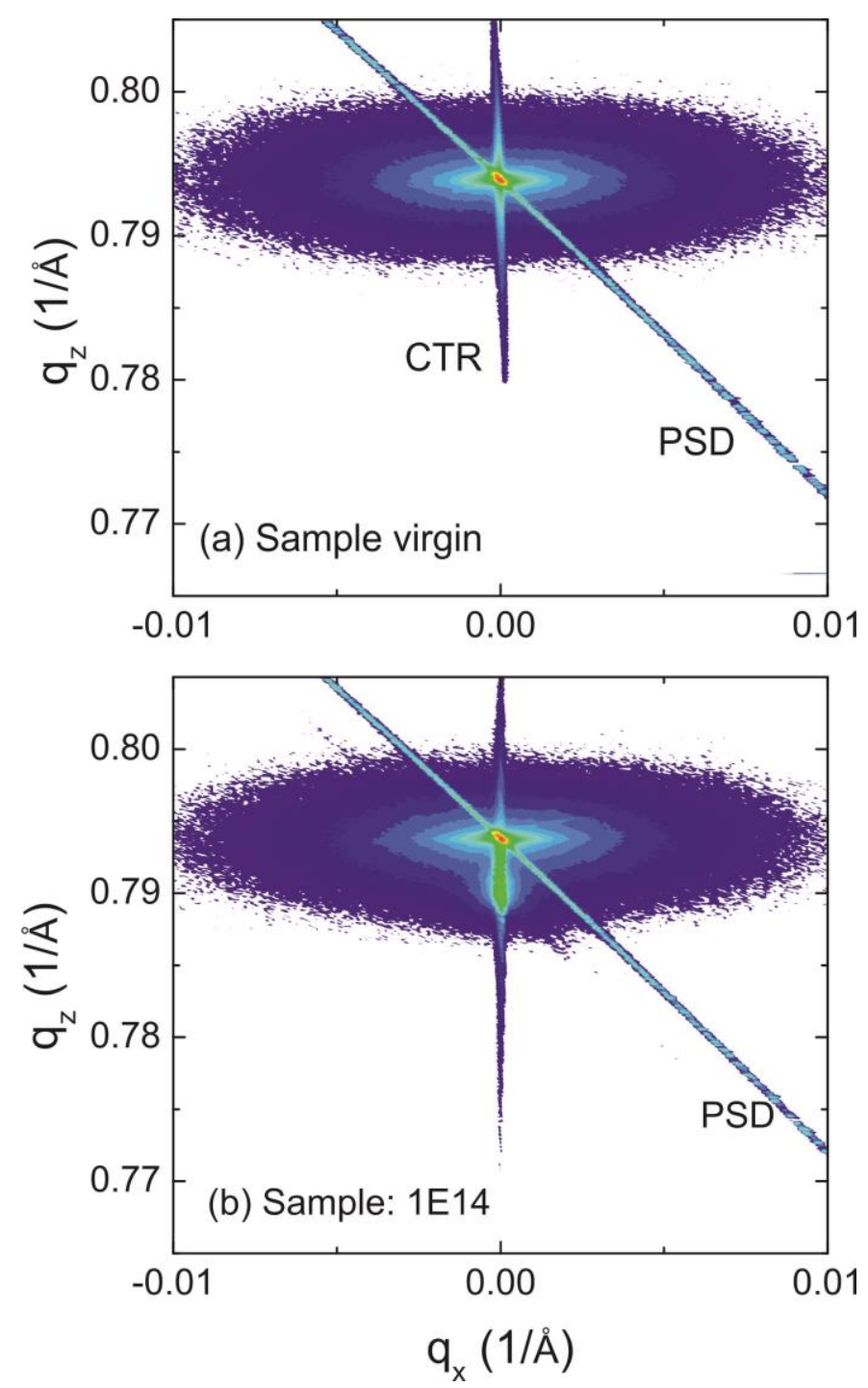

Fig. 4 Reciprocal space mapping of $\operatorname{SiC}(00012)$. There is no indication of broadening along $\mathrm{q}_{\mathrm{x}}$. The streak labeled CTR is the crystal truncation rod. The streak labeled PSD is due to the transmittance function of the position sensitive detector.

The RBS/C spectra along the SiC[0001] axis are shown in Fig. 5 for virgin and for $\mathrm{Ne}$ irradiated samples. The random spectrum is assumed equivalent to a completely amorphized $\mathrm{SiC}$, while the virgin spectrum corresponds to an essentially damagefree crystal. The minimum yield $\left(X_{\min }\right)$, the ratio of the channeling spectrum to the random spectrum, is $2.3 \%$ for the virgin $\mathrm{SiC}$ crystals at the surface region. For Page | 8 
sample 5E13 (not shown), the channeling spectrum is only slightly higher than the virgin sample. A peak in the damage depth around $150 \mathrm{~nm}$ is becoming visible for fluences of $5 \times 10^{14}$ and $1 \times 10^{15} / \mathrm{cm}^{2}$. Note that for sample $1 \mathrm{E} 15 \mathrm{Xmin}$ is $93 \%$, indicating the almost full amorphization at the damage peak. This is in agreement with the threshold displacement per atom (DPA) values for amorphization reported in literature [19]. DPA presents the irradiation effect considering both ion fluence and energy [20].

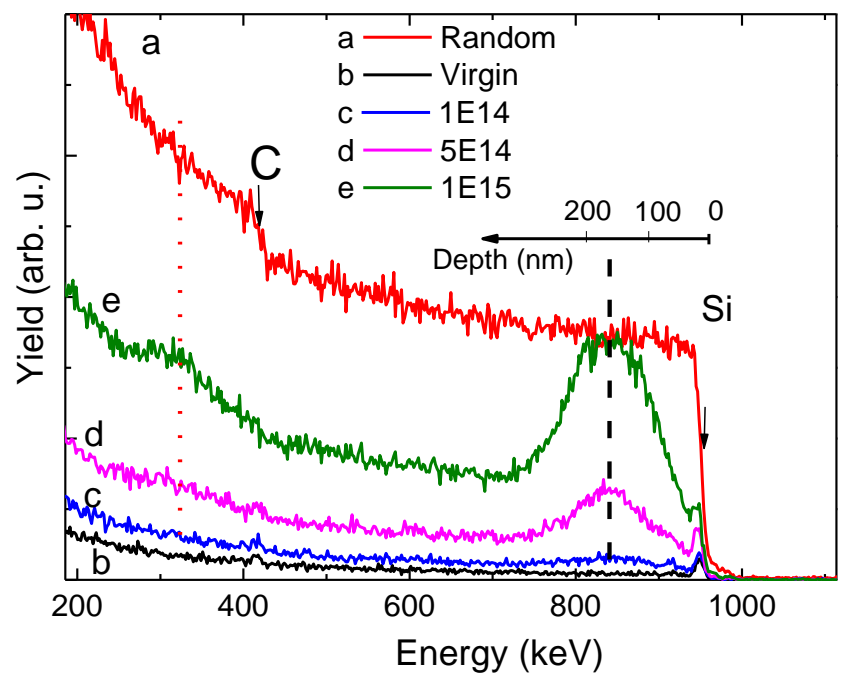

Fig. 5: RBS/C spectra for $6 \mathrm{H}-\mathrm{SiC}$ implanted with $\mathrm{Ne}$ ions. A random spectrum and a channeling spectrum from a virgin sample are also included for comparison. The dashed (dotted) line indicates the position of the damage peaks for $\mathrm{Si}$ (carbon). A rough depth scale is given.

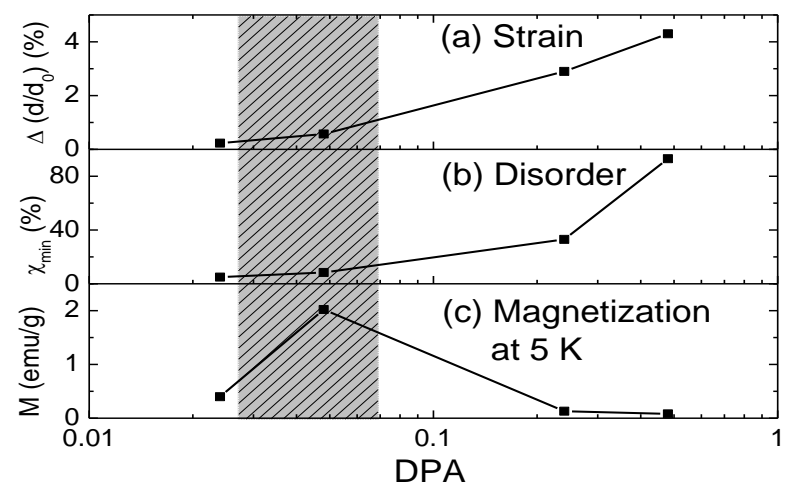


Fig. 6: Evolution of the saturation magnetization, the Si-sublattice disorder and the maximum strain with DPA. The gray area indicates the DPA range for optimizing the ferromagnetism.

\section{Summary}

Combining both structural and magnetic properties of ion irradiated $\mathrm{SiC}$, we are able to conclude a mutual relation between defect concentration and magnetization. As shown in Fig. 6, there is a narrow DPA window, within which the sample is ferromagnetic. The initial introduction of structural disorder (mainly point defects) leads to pronounced magnetization. Further increasing disorder induces agglomeration of point defects and finally amorphizes the host $\mathrm{SiC}$ crystal, consequently the ferromagnetism nearly drops to zero.

\section{Acknowledgement}

The work was financially supported by the Helmholtz Association (VH-NG-713). Y. Wang thanks the China Scholarship Council for supporting his stay at HZDR.

\section{Reference}

1. J. M. D. Coey, P. Stamenov, R. D. Gunning, M. Venkatesan, K Paul, New J. Phys. 12, 053025 (2010).

2. P. Esquinazi, D. Spemann, R. Höhne, A. Setzer, K.-H. Han, and T. Butz, Phys. Rev. Lett. 91, 227201 (2003).

3. Z. He, X. Yang, H. Xia, X. Zhou, M. Zhao, Y. Song, and T. Wang, Carbon 49, 1931 (2011). .

4. X. Yang, Z. He, W. Li, H. Xia, Y. Song, X. Zhou, X. Liu, M. Zhao, T. Wang, and K. Hou, J. Appl. Phys. 109, 083933 (2011).

5. J. Barzola-Quiquia, W. Böhlmann, P. Esquinazi, A. Schadewitz, A. Ballestar, S. Dusari, L. Schultze-Nobre, and B. Kersting, Appl. Phys. Lett. 98, 192511 (2011).

6. M. Venkatesan, C. B. Fitzgerald, and J. M. D. Coey, Nature 430, 630 (2004). 
7. Q. Xu, H. Schmidt, S. Zhou, K. Potzger, M. Helm, H. Hochmuth, M. Lorenz, A. Setzer, P. Esquinazi, C. Meinecke, and M. Grundmann, Appl. Phys. Lett. 92, 082508 (2008).

8. M. Khalid, M. Ziese, A. Setzer, P. Esquinazi, M. Lorenz, H. Hochmuth, M. Grundmann, D. Spemann, T. Butz, G. Brauer, W. Anwand, G. Fischer, W. A. Adeagbo, W. Hergert, and A. Ernst, Phys. Rev. B 80, 035331 (2009).

9. X. Zuo, S. Yoon, A. Yang, W. Duan, C. Vittoria, and V. Harris, J. Appl. Phys. 105, 07C508 (2009).

10. C. Araujo, M. Kapilashrami, X. Jun, O. D. Jayakumar, S. Nagar, Y. Wu, C. Århammar, B. Johansson, L. Belova, R. Ahuja, G. Gehring, and K. V. Rao, Appl. Phys. Lett. 96, 232505 (2010).

11. H. Thakur, P. Thakur, R. Kumar, N. B. Brookes, K. K. Sharma, A. P. Singh, Y. Kumar, S. Gautam, and K. H. Chae, Appl. Phys. Lett. 98, 192512 (2011).

12. C. Gómez-Polo, S. Larumbe, and J. M. Pastor, J. Appl. Phys. 113, 17B511 (2013).

13. L. Li, S. Prucnal, S. D. Yao, K. Potzger, W. Anwand, A. Wagner, and S. Zhou, Appl. Phys. Lett. 98, 222508 (2011).

14. Y. Liu, G. Wang, S. Wang, J. Yang, L. Chen, X. Qin, B. Song, B. Wang, and X. Chen, Phys. Rev. Lett. 106, 087205 (2011).

15. B. Song, H. Bao, H. Li, M. Lei, T. Peng, J. Jian, J. Liu, W. Wang, W. Wang, and X. Chen, J. Am. Chem. Soc. 131, 1376 (2009).

16. J. R. Jenny, D. P. Malta, S. G. Müller, A. R. Powell, V. F. Tsvetkov, H. M. D. Hobgood, R. C. Glass, and C. H. Carter: J. Electron. Mater. 32, 432 (2003).

17. P. Esquinazi, J. Barzola-Quiquia, D. Spemann, M. Rothermel, H. Ohldag, N. Garc'ia, A. Setzer, T. Butz, J. Magn. Magn. Mater. 322, 1156 (2010).

18. A. Debelle, L. Thome, D. Dompoint, A. Boulle, F. Garrido, J. Jagielski and D. Chaussende, J. Phys. D: Appl. Phys. 43, 455408 (2010).

19. W.J. Weber, L.M. Wang, N. Yu, and N.J. Hess, Mater. Sci. Eng. A 253, 62 (1998).

20. J. Ziegler, J. Biersack, and U. Littmark, The Stopping and Range of lons in Matter (Pergamon, New York, 1985). 
Fig. captions

Fig. 1: (a) Magnetization vs. field measured at $300 \mathrm{~K}$ for all samples: the diamagnetic background from the substrate has been subtracted. The magnetization was calculated by assuming a thin layer of 460 $\mathrm{nm}$ thickness. The inset shows the magnetization measured for the virgin sample (black line) and sample 5E13 without subtracting the background. (b) ESR spectra of sample $1 \mathrm{E} 14$ at temperatures between 100 and $300 \mathrm{~K}$ : a broad resonance peak appears indicating ferromagnetic resonance. The black line indicates the resonance centers which slightly change with temperature.

Fig. 2 PIXE spectrum for the virgin SiC wafer and a ferromagnetic sample. Within the detection limit, we do not observe any Fe, Co or Ni contamination.

Fig. $3 \mathrm{XRD} 2 \theta / \theta$ scans of the virgin and $\mathrm{Ne}$ irradiated $6 \mathrm{H}-\mathrm{SiC}$ recorded in the vicinity of the $(00012)$ reflection.

Fig. 4 Reciprocal space mapping of $\operatorname{SiC}(00012)$. There is no indication of broadening along $q_{x}$. The streak labeled CTR is the crystal truncation rod. The streak labeled PSD is due to the transmittance function of the position sensitive detector.

Fig. 5: RBS/C spectra for $6 \mathrm{H}-\mathrm{SiC}$ implanted with $\mathrm{Ne}$ ions. A random spectrum and a channeling spectrum from a virgin sample are also included for comparison. The dashed (dotted) line indicates the position of the damage peaks for $\mathrm{Si}$ (carbon). A rough depth scale is given.

Fig. 6: Evolution of the saturation magnetization, the Si-sublattice disorder and the maximum strain with DPA. The gray area indicates the DPA range for optimizing the ferromagnetism. 